\section{T3 SEVERE HYPOXIA EXISTS WITHIN PULMONARY TUBERCULOSIS LESIONS AND AUGMENTS MATRIX METALLOPROTEINASE-MEDIATED IMMUNOPATHOLOGY}

${ }^{1} \mathrm{M}$ Belton, ${ }^{1} \mathrm{~S}$ Brilha, ${ }^{2} \mathrm{~T}$ Fryer, ${ }^{2} \mathrm{Y}$ Hong, ${ }^{1} \mathrm{~F}$ Mauri, ${ }^{1} \mathrm{~N}$ Patel, ${ }^{3} \mathrm{~L}$ Tezera, ${ }^{1} \mathrm{~K}$ Nijran, ${ }^{3} \mathrm{PT}$ Elkington, ${ }^{1} \mathrm{JS}$ Friedland; 'Imperial College London, London, UK; ${ }^{2}$ Wolfson Brain Imaging Centre, Addenbrooks Hospital, University of Cambridge, Cambridge, UK; ${ }^{3}$ Department of Medicine, Southampton Hospital, Southampton, UK

\subsection{6/thoraxjnl-2013-204457.3}

Introduction Mycobacterium tuberculosis (MTb) causes approximately two million deaths each year. Extensive lung destruction is a hallmark of pulmonary tuberculosis and is caused by the breakdown of lung extracellular matrix by host matrix metalloproteinases (MMPs). Hypoxia upregulates gene expression and secretion of many inflammatory mediators via hypoxia-inducible factor (HIF-1 $\alpha$ ). Hypoxia has been demonstrated in several animal models of TB infection but no studies have been performed to investigate hypoxia in humans.

Methods To investigate the presence of hypoxia in human M.tb infection we performed PET-CT scanning using the hypoxia tracer [18F]FMISO in patients with pulmonary infection. Next, primary human monocyte-derived macrophages (MDMs) and human respiratory epithelial cells were stimulated by M.tb H37RV or conditioned media from Mtb-infected monocytes $(\mathrm{CoMTb})$ in a specially commissioned hypoxia workstation at 1\% O2, 5\% CO2. MMPs and their specific inhibitors (TIMP-1/ 2) were analysed by ELISA, Luminex array, zymography and confocal microscopy. Gene expression was analysed by qPCR and promoter activity by dual luciferase activity. Total HIF- $1 \alpha$ was measured by western analysis. The effect of HIF- $1 \alpha$ blockade was investigated using HIF-1a siRNA.

Results PET-CT scans demonstrated evidence of tracer trapping in regions of consolidation and in regions surrounding pulmonary cavities indicating severe hypoxia. Hypoxia potently upregulated MMP-1 in M.tb-stimulated primary MDMs and MMP-1 and MMP-9 in respiratory epithelial cells compared to normoxia $(\mathrm{p}<0.001)$. Similarly, hypoxia increased MMP-1 gene expression -2500 fold and potently increased MMP-1 promoter activity $(p<0.001)$. Site-directed mutagenesis of the NFKB binding site in the MMP-1 promoter and SC514 and helenalin chemical inhibition demonstrated that increased MMP-1 activity in hypoxia was regulated via an NFKB dependent mechanism. M.tb infection caused stabilisation of HIF- $1 \alpha$ protein even under conditions of normoxia but was more pronounced in hypoxia. Immunohistochemistry of patient samples demonstrated strong HIF-1 $\alpha$ immunoreactivity in tuberculosis patients compared to controls. HIF- $1 \alpha$ siRNA confirmed that increased MMP-1 activity in hypoxia occurred by a HIF- $1 \alpha$-dependent mechanism.

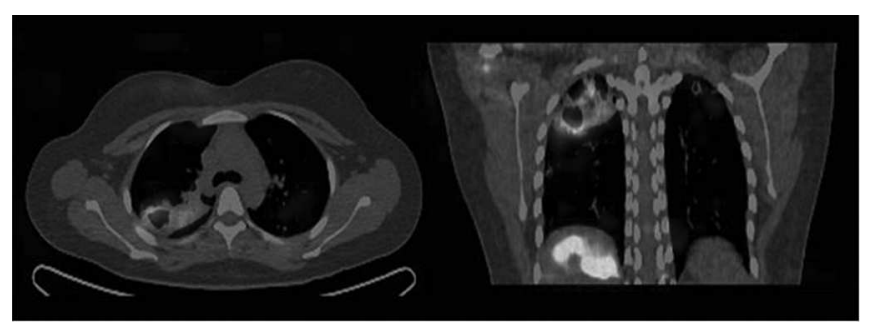

Abstract T3 Figure 1. Axial and coronal PET-CT images of a female patient with pulmonary $\mathrm{Tb}$. Trapping of the hypoxia tracer $18 F-F M I S O$ in TB-infected regions confirms severe hypoxia.

Conclusion We demonstrate for the first time that severe hypoxia develops within pulmonary TB lesions. Hypoxia increases
M.tb-driven MMP-1/9 gene expression and secretion in a HIF1a-dependent manner. These results support the hypothesis that during $\mathrm{Tb}$ infection, hypoxia develops and promotes immunopathology.

\section{T4 A CROSS-SECTIONAL ANALYSIS OF THE EFFECT OF COPD ON WORK CAPABILITY USING THE BIRMINGHAM COPD COHORT}

${ }^{1} \mathrm{~K}$ Kalirai, ${ }^{2} \mathrm{P}$ Adab, ${ }^{2} \mathrm{R}$ Jordan, ${ }^{2} \mathrm{D}$ Fitzmaurice, ${ }^{1} \mathrm{~J}$ Ayres; ${ }^{1}$ Institute of Occupational and Environmental Medicine, The University of Birmingham, Birmingham, England; ${ }^{2}$ Public Health, Epidemiology \& Biostatistics, The University of Birmingham, Birmingham, England

\subsection{6/thoraxjnl-2013-204457.4}

Introduction The effect of COPD on work is poorly understood. Approximately $40 \%$ are of working age, however, employment rates are lower compared to others. Little is known about characteristics of COPD patients who remain in employment compared to those who do not.

Aim To assess which factors are associated with employment status among working age COPD patients.

Methods 2000 patients with COPD from primary care are being recruited into a three-year cohort study. In addition to clinical data, occupational history and work performance are assessed. Interim baseline data was used to assess associations between employment status, co-morbidities, COPD assessment test (CAT) scores (impact of COPD on HRQL), lung health, BMI and exercise capacity among COPD patients of working age.

Results Of the 1094 patients recruited $31.8 \%(\mathrm{n}=348)$ were of working age (mean age 54.9), of whom 31.6\% $(\mathrm{n}=110)$ were in work. Compared to those not in work, working patients were more likely to be male $(72.7 \%$ vs $52.5 \%)$ but were similar in terms of smoking history (ever smokers: $94.4 \%$ vs $91.3 \%$ ).

Overall, working patients had fewer co-morbidities. Adjusted for age, sex and smoking status, they were less likely to have cardiovascular disease $(\mathrm{OR}=0.58 ; 95 \%$ CI $0.38-0.87)$, gastrointestinal disease $(\mathrm{OR}=0.37 ; 0.20-0.69)$, cancer $(\mathrm{OR}=0.09$; $0.01-0.72)$ ) and osteoarthritis $(\mathrm{OR}=0.4 ; 0.19-0.84)$. Being in work was associated with less dyspnoea $(\mathrm{OR}=0.12 ; 0.05-0.25$ for MRC grade 1 vs. grade 5), higher quality of life $(\mathrm{OR}=0.34 ; 0.14-0.85$ for low vs. very high CAT score $)$ and a greater exercise capacity $(\mathrm{OR}=1.14 ; 1.08-1.19)$, but BMI was not associated with employment status $(\mathrm{OR}=1.0 ; 0.97-1.06)$. There was no difference in disease severity (assessed by GOLD stage) between the two groups ( $p$ for trend $=0.16$ ).

Conclusions This is the first primary care study in the UK to consider the impact of COPD on work capability. COPD patients who are in work tend to be healthier, are less breathless and have a better quality of life than those not in work, even though disease severity in the two groups was similar. The findings from this analysis suggest that being in work is good for health but longitudinal studies are needed to confirm this.

\section{T5 CIRCULATING ENDOTHELIAL PROGENITOR CELLS IN SMOKERS AND PATIENTS WITH COPD ARE DYSFUNCTIONAL DUE TO INCREASED DNA DAMAGE AND SENESCENCE}

${ }^{1} \mathrm{~K}$ Paschalaki, ${ }^{2} \mathrm{RD}$ Starke, ${ }^{3} \mathrm{Y}$ Hu, ${ }^{1} \mathrm{~N}$ Mercado, ${ }^{3} \mathrm{~A}$ Margariti, ${ }^{4} \mathrm{VG}$ Gorgoulis, ${ }^{2} \mathrm{AM}$ Randi, ${ }^{1} \mathrm{PJ}$ Barnes; ${ }^{1}$ Airway Disease Section, National Heart and Lung Institute, Imperial College London, London, United Kingdom; ${ }^{2}$ Vascular Science, National Heart and Lung Institute, 